REVISTA ECONOMÍA

Vol. 7I, N. ${ }^{\circ}$ II3 (mayo 20I9), 63-74

\title{
IMPASSE LATINOAMERICANO. LÍMITES DEL PATRÓN EXPORTADOR DE ESPECIALIZACIÓN PRODUCTIVA
}

\author{
JOSÉ LUIS RÍOS \\ Universidad Nacional Autónoma de México, UNAM
}

Recepción de manuscrito: 26 de abril de 2019

Aceptación de versión final: 30 de abril de 2019

\begin{abstract}
RESUMEN Este trabajo analiza algunos elementos relevantes de la categoría patrón de reproducción del capital. Dentro de este referente teórico, se estudia la categoría de patrón exportador de especialización productiva, la cual designa la modalidad de reproducción del capital actualmente vigente en la región latinoamericana y un modo de integración en la división internacional del trabajo. El trabajo expone las bases del patrón exportador, señala sus caracterizaciones principales y sus limitaciones. Por último, se propone estudiar el declive y crisis de este patrón de reproducción en relación con la crisis del capitalismo mundial, y advierte sobre el impasse en el que se encuentra la región.
\end{abstract}

PALABRAS CLAVE Patrón de reproducción del capital, patrón exportador de especialización productiva, crisis del patrón exportador.

ABSTRACT In this paper we analyze some important elements of the pattern of reproduction of the capital category. Within this theoretical framework, we study the category of exporting pattern of productive specialization, which designates the modality of reproduction of the capital currently in force in the Latin American region and a mode of participation in the international division of labor. We expose the bases of the exporting pattern, we indicate its main characterizations and its limitations. Finally, we study the decline and crisis of this pattern of reproduction in relation to the crisis of world capitalismo, and we warned about the impasse in which the region is located.

KEYWORDS Pattern of reproduction of capital, exporting pattern of productive specialization, crisis of the exporting pattern.

\section{INTRODUCCIÓN}

En el presente trabajo son estudiados algunos de los elementos relevantes sobre la categoría de patrón de reproducción del capital. Esta categoría adquiere centralidad en la investigación sobre las formas históricas que asume el proceso de valorización del capital en la región latinoamericana. Bajo este marco teórico, se analiza la categoría de patrón exportador de especialización productiva, la cual designa la modalidad de reproducción del capital actualmente vigente en 
la región. A la luz de la dinámica que ha asumido el patrón exportador, se reflexiona sobre el lugar de América Latina en la actual división internacional del trabajo y las dificultades que atraviesa. El artículo elabora un cuadro crítico de las bases del actual patrón exportador, mostrando las razones de su aceleración fundamentalmente en la primera década del 2000. Además de exponer sus caracterizaciones principales, se subrayan sus limitaciones. Por último, se estudia el declive y crisis de este patrón de reproducción del capital, que se sucede en relación a la crisis del capitalismo mundial, lo que coloca a la región en un enorme impasse del que solo es posible salir si se cuestionan las bases de integración dependiente al capitalismo mundial.

\section{SOBRE LA CATEGORÍA DE PATRÓN DE REPRODUCCIÓN DEL CAPITAL}

En esencia, el capital tiene por principio y finalidad su valorización. Para tal objeto, da vida a un movimiento cíclico constituido por tres fases: circulación, producción, circulación. En cada una de éstas, el capital asume distintas formas bajo las que deberá reunir una serie de condiciones que le permitan acceder a su objetivo final de valorización-acumulación.

La categoría de patrón de reproducción del capital ${ }^{1}$ permite hacer inteligible el modo particular en que el capital realiza su proceso de valorización. Las formas que adoptan las relaciones construidas por el capital y las modalidades que éste asume en el flujo de la circulación y de la producción - por ejemplo, origen y montos de inversión, origen y tipo de tecnología incorporada, modo de consumo de fuerza de trabajo, composición de mercados-, ofrecen luces para entender el singular movimiento que el capital adopta en sus distintos procesos y esferas en un tiempo y espacio dados. La dinámica de sus movimientos y relaciones adoptadas en cada una de sus fases se vuelven asequibles bajo los caracteres de lo que se denomina patrón de reproducción.

La fórmula que permite la producción de conocimientos sobre el patrón de reproducción capitalista es obtenida mediante el conocimiento del ciclo del capital-dinero, el cual fue desarrollado por Karl Marx, el libro II, primera sección, de su obra magna y se expresa en la siguiente formulación: D-M... P... M M'-D'. El conjunto de relaciones presentes en cada una de las fases de este ciclo son susceptibles de tratamiento analítico, de ahí que la categoría de patrón de reproducción ofrece una riqueza teórica y metodológica con la que se vuelven asequibles las distintas relaciones y procesos que el capital construye en su movimiento cíclico de reproducción.

En el marco de un sistema capitalista mundial, estudiar el patrón de reproducción del capital implica investigar la reproducción del capital dentro de las relaciones entre economías centrales y dependientes que constituyen al sistema heterogéneo y diferenciado del capitalismo mundial. Dentro de este marco, permite explicar la especificidad original de los modos de reproducción establecidos en regiones y formaciones sociales dadas. Comprender esto, supone reconocer la importancia que tiene la operación de mecanismos de transferencias de valor intercambio desigual; transferencias de ganancias a los países sede de las corporaciones trasnacionales; pago de intereses vía deuda externa, deuda pública; renta tecnológica; procesos de desacumulación; etc.- , conformados por las relaciones de explotación internacional que menoscaban a las economías dependientes. 
Asimismo, estudiar la reproducción del capital no supone el análisis de «lo económico» de un modo aislado e independiente de otras relaciones sociales, sino que implica de modo indivisible el estudio complejo de las relaciones políticas que le subyacen. El poder político y sus relaciones de dominio siempre han acompañado los procesos de desposesión que dieron origen a las relaciones sociales capitalistas. ${ }^{2}$ En el desenvolvimiento histórico de estas relaciones capitalistas - apropiación del trabajo no retribuido, reproducción del asalariado, precarización del trabajo, etc.-, las relaciones de coerción y de dominación política no dejan de estar presentes (Poulantzas, 1976, pág. 90).

De este modo, a partir de la categoría de patrón de reproducción pueden estudiarse relevantes procesos referidos al papel que asume lo político en el movimiento de reproducción del capital, por ejemplo, (a) el lugar del Estado en el patrón de reproducción; (b) el papel económico del aparato estatal; y (c) la configuración de relaciones y alianzas políticas (bloque en el poder) así como las relaciones de hegemonía, etc. Sobre este referente teórico-analítico, a continuación se reflexiona sobre la vigente forma de reproducción del capital que se ha adoptado en América Latina.

\section{LA CATEGORÍA DE PATRÓN EXPORTADOR DE ESPECIALIZACIÓN PRODUCTIVA}

\section{GENERALIDADES Y PARTICULARIDADES DEL PATRÓN EXPORTADOR}

Desde finales de siglo xx, América Latina se asienta en la construcción de un nuevo patrón de reproducción del capital exportador de especialización productiva. Con la categoría de patrón exportador de especialización productiva ${ }^{3}$ se designa una forma particular que el capital construye en las distintas esferas, fases y procesos que constituyen a su ciclo general. Cinco de sus principales rasgos definen su caracterización integral: (a) el papel que adopta el mercado externo como fundamento central en la realización del capital; (b) una concentración privilegiada de determinados y reducidos ejes y ramas productivas en la esfera de la producción con efectos desfavorables en la estructura industrial; (c) remarcada significación de las relaciones de explotación y superexplotación del trabajo; (d) estrechas relaciones entre las clases dominantes locales con el capital internacional; (e) un relevante papel del Estado en su forma de reproducción.

El patrón exportador de especialización productiva es la modalidad vigente que adopta el movimiento de circulación y reproducción del capital en América Latina. El hecho de que este patrón exportador constituya el fundamento del ciclo del capital en la región latinoamericana, permite hablar de importantes caracteres análogos entre las economías de la región. No obstante, los estudios sobre la reproducción del capital ponen de relieve la necesidad de profundizar sobre las particularidades y singularidades que caracterizan a nuestras economías. Fuentes de inversión conformadas, sectores productivos receptores de éstas, actividades productivas realizadas, tipo de valores de uso elaborados, espacios geográfico-productivos constituidos, composición de mercados, etc., permiten investigar relaciones y configuraciones de especificidad propia de cada una de las formaciones sociales de la región, las que se hacen aún más originales al conocer la complejidad de sus caracterizaciones políticas inherentes. 
PATRÓN EXPORTADOR LATINOAMERICANO Y DIVISIÓN INTERNACIONAL DEL TRABAJO

Las principales limitaciones de la economía latinoamericana respecto a su integración subordinada a la mundialización del capital, pueden entenderse en el marco de las nuevas cadenas de valor global que dan forma a la división internacional del trabajo. Esta división adquirió mayor significación y profundización a raíz de la gran convulsión que sufrieron las relaciones espacio/tiempo, la cual fue auspiciada por la revolución tecnológico-científica - en sectores de la informática, microelectrónica, telecomunicaciones, etc.- - Dicha revolución promovió en las últimas décadas de siglo xx una radical reestructuración de las relaciones sociales de producción de alcance global, la cual estableció procesos de segmentación de las actividades productivas y nuevos encadenamientos para la producción de valor. Se implementaron nuevos procesos de reestructuración de fases productivas y una fragmentación de eslabones relocalizados en países de cualquier región del mundo, lo cuales fueron llevados a cabo bajo la dirección de la gran empresa trasnacional.

Esta reorganización global de la producción se ha establecido mediante los más violentos impactos en la gestión científica del capital y de la fuerza de trabajo (modelos posfordistas, toyotismo) produciendo una reestructuración del mundo del trabajo a escala global que ha destacado por sus cambios profundos en cuanto a los modos de conformación, distribución y (des) organización del trabajo; desregulación de mercados de trabajo; niveles de retribución salarial; mecanismos de seguridad y representación (precarización laboral); extensión e intensificación del trabajo (formas de explotación); calificación/descalificación de la fuerza laboral (Sotelo, 2003; Antunes, 2005).

La internacionalización de los procesos de trabajo se ha caracterizado por el control de los países centrales y el comando de la empresa trasnacional. Dichos países concentran el monopolio tecnológico y centralizan los mayores niveles de inversión en ciencia y tecnología. Al mismo tiempo, dislocan (no sin contradicciones) hacia los países atrasados y periféricos sus actividades productivas con menor intensidad tecnológica, lo que robustece en los países de la región los problemas del subdesarrollo. Esta forma de integración de los países de la región latinoamericana a la economía mundial, alienta la creación de espacios y actividades de especialización productiva, con el establecimiento de una economía sustentada en recursos naturales. El principal efecto de la especialización es el deterioro de un conjunto de relaciones productivas, o incluso su propio desmantelamiento, que es lo que explica los graves procesos de desindustrialización que experimenta la región. ${ }^{4}$

El vigente estado de la división internacional del trabajo ha llevado a una mayor acentuación de las asimetrías existentes entre los países centrales y los países dependientes. Dichas asimetrías se expresan para los países dependientes en la acentuación de su heterogeneidad productiva y sus desequilibrios estructurales, lo que pone de relieve los límites de nuestros países para acceder al «desarrollo».

Otra de las contradicciones que sobresalen de la conformación de la división global del trabajo, se refiere a la generación de mayores obstáculos para las economías subdesarrolladas. Los nuevos encadenamientos de la producción refuerzan los obstáculos que enfrentan las economías nacionales para articular sectores, eslabones y encadenamientos productivos. Esta debilidad estructural, heredada desde décadas atrás, es agravada e impacta de modo progresivo en 
formas de articulación-desarticulación geográfico-territorial de las economías nacionales (desterritorialización), con fuertes impactos sociales (de clase, raciales, electorales). Esta tendencia a la desarticulación productiva y a la concentración de la economía en unos cuantos sectores y espacios productivos de especialización, se ve fortalecida con el creciente poder económico de los grandes oligopolios trasnacionales (industriales, energéticos, agromineros, de servicios, financieros), lo que conduce a una mayor acentuación de las problemáticas relaciones de soberanía - y su relación con la democracia - que caracterizan a los países de la región, problemas que inciden en la incapacidad de elaborar y establecer patrones y vías de desarrollo «nacional», así como estrategias de integración regional.

AMÉRICA LATINA EN LA NUEVA DIVISIÓN INTERNACIONAL DEL TRABAJO

Dentro de este cuadro, es importante reflexionar sobre la conformación de una renovada jerarquización en el nuevo estadio de la división internacional del trabajo, vinculada a la cuarta revolución tecnológico-industrial, la cual es impulsada mediante los dominios de la industria 4.0, la inteligencia artificial, robótica, semiconductores, automóviles autónomos, eléctricos, red 5G. En este contexto, ¿cuál es el lugar que ocupará América Latina en el nuevo estadio de la producción internacional?

Ante ello, es relevante considerar la debilidad de las capacidades de inversión y desarrollo tecnológico de la región. De acuerdo con la CEPAL (2017), en materia de capacidades tecnológicas, América Latina y el Caribe concentran el 2\% de las solicitudes mundiales de patentes en 1990. Veinticinco años después, en el año de 2015, su participación es igualmente del 2\%. En contraste, en los mismos años, Estados Unidos pasa del 17 al 20\%; China asciende de modo espectacular del 1\% al 38\%; Japón desciende vertiginosamente del 37 al 11\%; Europa cae del 35 al 12\%. En resumen, China, Alemania y Estados Unidos lideran las capacidades tecnológicas en dichas solicitudes. ${ }^{5}$

Las economías centrales, al monopolizar fases y paquetes tecnológicos dirigidos a valores de uso con alta intensidad de conocimiento, reproducen de un modo agresivo los mecanismos de intercambio desigual entre las economías. En contraste, valores de uso correspondientes a sectores especializados y ramas de bienes primarios, industria extractiva, alimentos y materias primas con bajos niveles de transformación, si bien experimentan de modo coyuntural la elevación de sus precios, en el largo plazo, estos retoman su tendencia decreciente, lo que acentúa el deterioro de los términos de intercambio e incide en procesos de transferencias de valor y apropiación de renta, con efectos en la mayor explotación del trabajo. ${ }^{6}$

Frente al nuevo estado de la cuarta revolución tecnológico-industrial y las condiciones de monopolio tecnológico, la economía latinoamericana no puede sustentar sus modalidades de reproducción económica basadas en un abastecimiento de bienes tradicionales, o, en su caso, especializadas en un número reducido de actividades productivas. ${ }^{7}$ Más de dos décadas de especialización de actividades productivas volcadas a los mercados externos han mostrado las repercusiones negativas en el aparato productivo, escenarios desfavorables de los términos de intercambio, aumento de transferencias de valor al exterior, y por ende, la exacerbación de las condiciones de superexplotación del trabajo que fortalecen las bases de reproducción dependiente de la economía latinoamericana (Marini, 1974). 


\section{LAS BASES DEL PATRÓN EXPORTADOR DE ESPECIALIZACIÓN PRODUCTIVA}

En la primera década de 2000, América Latina radicaliza el abandono de los procesos de diversificación industrial dominantes en el periodo 1940-1980 y vuelca su estructura económica hacia la consolidación del patrón exportador de especialización productiva. Al configurar de modo selectivo a ciertos sectores y actividades económicas - la mayor parte de ellos con «ventajas naturales»- como polos dinámicos o núcleo de valorización del capital, lo hace en desmedro de una articulación compleja y diversificada del aparato productivo.

EL BOOM EXPORTADOR: VALORES DE USO TRADICIONALES Y ESPECIALIZACIÓN PRODUCTIVA

Excluyendo la desaceleración de las exportaciones mexicanas en el periodo 2000-2009, la tasa media anual de crecimiento de las exportaciones de bienes de la región pasa de 5,4\% en la década de 1990 a 9,8\% el periodo 2000-2009 (CEPAL, 2011-2012). Respecto con América del Sur, esta subregión ve duplicar su tasa anual de crecimiento de las exportaciones de 5,2\% a 10,6\%. Si analizamos el desglose y descomposición de las exportaciones totales, tanto las que están basadas en recursos naturales como sus distintos grados de elaboración, destaca el proceso de primarización de las exportaciones de la región.

Al desagregar las exportaciones de manufacturas en sus componentes principales, sea por intensidad tecnológica incorporada — basadas en recursos naturales, en tecnología baja, en media o alta tecnología-, o sea por intensidad del uso de factores - en ingeniería, en recursos naturales, en trabajo-, se comprueba que el mayor peso de las exportaciones manufactureras de la región en la década de 2000 reside en la mayor intensidad en recursos naturales, lo que fortalece el cuadro de la especialización productiva centrada en la exportación de productos primarios.

El ascenso de commodities e insumos industriales (minerales, siderúrgicos, alimentos, celulosa, agrocombustibles y energía) exportados tanto a nivel regional como por país, les permite constituirse como el sustento de la especialización productiva. Bolivia, Ecuador, Chile, Perú y Paraguay concentraron en el año de 2009 alrededor del 85\% de las exportaciones en estas mercancías. Países como Brasil, Uruguay, Argentina, Colombia, concentraron alrededor del $60 \%$ de sus exportaciones en commodities. En contraste, se observa el caso de México que concentró en este mismo año el $24 \%$ de sus exportaciones en commodities, lo que se explica por el peso de la industria maquiladora en su estructura exportadora.

\section{CAUSAS DEL BOOM Y MEJORA RELATIVA Y COYUNTURAL EN LOS TÉRMINOS DE INTERCAMBIO}

El dinamismo exportador que marcó el auge de los productos básicos en la década de 2000, se explica en razón del crecimiento de los precios de los principales productos de los países de la región (con niveles máximos históricos en 2008), fundamentalmente en los países productores de petróleo, cobre, mineral de hierro, acero, gas natural, soya, carne, café. ${ }^{8}$ Dicha elevación de los precios fue vinculada a las condiciones de la producción y demanda global de aquella década, al papel de China con su enorme demanda de materias primas, así como a las crecientes relaciones sino-latinoamericanas. Así, es necesario subrayar que la tasa de crecimiento tanto de China como del mundo en el período 2000-2007 no sería la misma para el periodo poscrisis a partir de 2009. 
En el periodo 2000-2008 de crecimiento de los precios en los productos básicos, la región registró una mejora en los términos de intercambio de alrededor del 20\% (CEPAL, 2009-2010, pág. 78). Chile y la Comunidad Andina destacaron por su mayor aumento en la relación de intercambio de alrededor de $64 \%$ y $73 \%$, respectivamente. Los países del Mercosur y México registraron aumentos menores. En cambio, los países de Centroamérica, al ser importadores netos de alimentos y combustibles, sufrieron un deterioro del intercambio de $18 \%$.

\section{SUPERÁVITS, POLÍTICAS SOCIALES Y RENUNCIA A LA TRANSFORMACIÓN}

El conjunto de estos elementos señalado marcaron el boom exportador con el que la región profundizó su integración a la economía mundial. Fue el auge de la especialización exportadora lo que llevó a los mega superávits comerciales que favorecieron a los gobiernos «progresistas» de la región, e incluso a los no progresistas (Chile, Perú, Colombia). De esta manera, se construyó un modelo que fue denominado como «crecimiento económico con distribución de renta», con mucha mayor fuerza en los gobiernos señalados.

No obstante puede señalarse que durante el periodo de bonanza económica ${ }^{9}$ no fueron construidas nuevas capacidades productivas, que impulsaran sectores intensivos en conocimiento, innovación, o nuevos sectores tecnológicos. No se acortó la distancia tecnológica o de productividad con las economías centrales. No se pusieron en cuestionamiento las bases estructurales de dependencia de la economía latinoamericana.

Se llevaron a cabo transferencias de renta (a través del Estado), esto es, sobre la base de las mismas condiciones de concentración de la riqueza y mantenimiento de los privilegios. ${ }^{10}$ En dicho periodo, estas bases de concentración económica no fueron removidas. Disminuyó relativamente la pobreza, no así la desigualdad. Más aun, se acentuó la concentración del ingreso, y ello acorde a la modalidad de acumulación hegemonizada por los grandes ganadores de este patrón de reproducción: la gran empresa exportadora trasnacional. ${ }^{11}$

Durante la primera década de 2000, la región experimentó un crecimiento en el PIB. Sin embargo, no debe dejarse de lado que lo consiguió sobre una dinámica económica que acentuaba sus condiciones de dependencia, esto es, respecto a los ingresos provenientes de exportaciones tradicionales, y aceptando un lugar subordinado en la división internacional del trabajo. En este sentido, los procesos de especialización productiva de la región consolidaron una división internacional del trabajo marcada por una regresión a la especialización exportadora de finales de siglo xix. No obstante, su característica distintiva reside en su conformación actual dentro del nuevo estadio de internacionalización tecnológica de los procesos de trabajo, el dominio hegemónico del capital financiero y el ascenso de China en el concierto mundial.

En resumen, con la especialización latinoamericana sustentada en recursos naturales y su incorporación a las cadenas de valor, el patrón de especialización productiva renunció a un proceso de acumulación orgánica, con mayores equilibrios y niveles de integración entre sus sectores; deterioró el aparato productivo en su capacidad de producir con los mayores grados de elaboración y generación de valor, al tiempo que lo sustentó en la mayor explotación al mundo del trabajo reflejada con el aumento de la desigualdad social. Más allá de relativos y coyunturales avances logrados, puede decirse que se desarrolló el subdesarrollo. 


\section{CRISIS DEL PATRÓN EXPORTADOR}

Contrario al periodo $2000-2007$, los años posteriores a 2008 experimentaron un descenso de los precios en los productos primarios, mientras que los precios de manufacturas se han mantenido estables. No obstante, la disminución en el ritmo de crecimiento de la economía China derivada de la crisis financiera de 2008, implicó el descenso de sus exportaciones de manufacturas y, por ende, la caída de su demanda de productos tradicionales. ${ }^{12}$

En el cuatrienio 2013-2016, América Latina experimentó un fuerte desplome en el valor de sus exportaciones (2013: -0,4\%; 2014: -3\%; 2015: -14\%; 2016: -3,2\%). ${ }^{13}$ En estos años, la profundización de la incertidumbre global logró quebrar las políticas anticíclicas que algunos países dependientes de commodities implementaron, incluso, ensayando modificaciones del motor externo del dinamismo económico al motor interno. La economía latinoamericana nuevamente se encontró con los problemas en la balanza comercial y en la balanza de pagos, y se agravó así el marco de la restricción externa para lograr crecimiento, más aún, con el creciente endeudamiento de las economías. En el último lustro, de acuerdo con datos de CEPAL (2016; 2018), la economía latinoamericana ha presentado recesión (año 2015: -0,5\%; 2016: -1,1\%) y cuasi estancamiento económico (año 2014: 0,9\%; 2017: 1,2\%; 2018: 1,3\%). Puede señalarse que en el bienio 2017-2018 la región logró una recuperación en el valor exportado (2017: 11,5\%; 2018: 9,7\%) (CEPAL, 2018). No obstante, una vez más se trata de una recuperación dependiente del incremento inestable de los precios en commodities, que en estos dos años fue de 7 y $7,6 \%$, respectivamente. En contraste, se experimentó un crecimiento débil en el volumen exportado (alrededor de $2 \%$ ).

Los mayores riesgos para la región en los años 2019 y 2020 son la desaceleración comercial que podría reducir los precios de productos básicos, y por ende, llevar a la caída en el valor de las exportaciones. Al mismo tiempo, el incremento de las tensiones comerciales propiciadas por el enfrentamiento de Estados Unidos con China, podrá inducir a una mayor inestabilidad e incertidumbre en los mercados financieros, presionando a las inversiones y sus impactos en las cadenas de suministro con efectos en los precios de las materias primas.

Bajo estos parámetros de inestabilidad y atraso, ¿puede la región mantenerse sobre estas condiciones de reproducción del capital? Más aun, señalemos que la crisis mundial de nuestro tiempo está vinculada a la crisis estructural del capital (Mészaros, 2009). La crisis actual del sistema mundial del capital marcado por el llamado «estancamiento de larga duración» (Bellamy Foster y F. Magdoff, 2010), viene secando todo ensayo dirigido a la expansión del crecimiento. La lógica que envuelve a la crisis mundial hace muy difícil la recuperación (Amín, 2016).

En las condiciones actuales de la economía mundial, se registra, por un lado, el predominio destructivo del capital financiero, el sistema de austeridad y el excesivo endeudamiento global muy próximo al 300\% del PIB mundial. Por el otro lado, se registra el descenso del comercio mundial, la presión sobre la inversión, sobre la demanda y sus consecuencias en el crecimiento con riesgos incluso de recesión económica global (ver Tabla 1). Dentro de este marco, se inscriben las tendencias del America First de D. Trump, su denodada política de liberalización financiera, su guerra tecnológica y comercial contra la economía China, procesos que junto a los crecientes conflictos geopolíticos alrededor del mundo, agravan las condiciones de inestabilidad económica global. Las estimaciones para 2020 anuncian una recesión 
Tabla 1: Economía mundial (varios países y AL): tasas de crecimiento del PIB y del comercio mundial

\begin{tabular}{|c|c|c|c|c|c|c|c|}
\hline & 2013 & 2014 & 2015 & 2016 & 2017 & 2018 & $2019^{*}$ \\
\hline Mundo & 2,3 & 2,6 & 2,4 & 2,5 & 3,2 & 3,3 & 3,2 \\
\hline Estados Unidos & 1,5 & 2,4 & 2,4 & 1,5 & 2,3 & 2,8 & 2,3 \\
\hline Japón & 1,6 & $-0,1$ & 0,5 & 0,9 & 1,7 & 1,1 & 1 \\
\hline Euro zona & $-0,3$ & 0,9 & 1,7 & 1,8 & 2,4 & 2,2 & 1,9 \\
\hline Rusia & 1,3 & 0,6 & $-3,7$ & $-1,9$ & 0,6 & --- & --- \\
\hline China & 7,7 & 7,3 & 6,9 & 6,7 & 6,9 & 6,6 & 6,3 \\
\hline América Latina & 2,9 & 0,9 & $-0,5$ & $-1,1$ & 1,2 & 1,3 & 1,3 \\
\hline Comercio global & 2,4 & 2,4 & 2,7 & 1,5 & 4,6 & $3^{* *}$ & $2,6^{* *}$ \\
\hline
\end{tabular}

Fuente: Elaborado con datos de Cepal, Estudio económico de AL y el Caribe $(2016,2018) ;{ }^{\star}$ Estimados de Cepal; ${ }^{\star *}$ Estimados OMC (2019).

en la economía de Estados Unidos.

En el desenvolvimiento de los problemas de inestabilidad económica global, la región latinoamericana se enfrenta al agravamiento de las contradicciones a nivel de las relaciones internacionales de explotación. La complicación del escenario internacional en las relaciones comerciales, productivas y financieras - sin ignorar la guerra económica del imperialismo a las economías latinoamericanas, como lo experimenta Venezuela, Cuba, Nicaragua-, la incesante búsqueda de materias primas y alimentos, representan una seria afrenta para los países dependientes, más aún, con la vuelta de la irrupción del unipolarismo militarista de Estados Unidos en el concierto geopolítico global. El cuadro crítico del imperialismo empuja a un nuevo nivel de agresión sobre los países dependientes, la que es más acentuada en la región, dadas las crecientes relaciones de América Latina con la economía de China, Rusia, Irán e India, quienes al representar nuevos polos del poder mundial, desafían la hegemonía (en declive) de Estados Unidos.

Bajo esta dinámica de inestabilidad y crisis en curso, adquieren su mayor relevancia los procesos de intercambio desigual y transferencias de valor que experimentan las economías dependientes. Como ya señalamos, sobre las bases de la especialización productiva, son acentuados estos mecanismos de explotación internacional entre las economías. Su principal impacto lleva a reproducir en otra escala los rezagos de la región y sus principales contradicciones estructurales.

\section{CONCLUSIÓN}

En el curso actual de la economía global, todo indica que la región se encuentra en un enorme impasse. Bajo el despliegue de esta «crisis interminable» del capital, se imponen a nuestra región enormes desafíos. Sus modalidades de integración al capitalismo mundial reproducen ciegamente un papel subordinado en los procesos de producción-circulación y acumulación-desacumulación promovidos por una asociación entre clases dominantes locales y extranjeras. El desafío implica el cuestionamiento a las condiciones de adaptación a la economía mundial, que las experiencias recientes en la región llevaron a cabo sin romper con los nudos de dependencia de nuestros países, actualmente atados a un patrón de reproducción 
que a más de dos décadas de vigencia, ha alcanzado su propio agotamiento, paralelo a la crisis histórica del sistema capitalista mundial.

El desafío principal apunta hacia la construcción de necesarias mudanzas en sus modalidades de reproducción que promuevan otras condiciones de vida fundadas en relaciones de soberanía, autodeterminación, democracia e igualdad sustantivas. Precisamente, lo que la lógica del capitalismo mundial, hoy menos que nunca, puede ofrecer a nuestras sociedades.

\section{NOTAS}

1 Esta categoría se remonta a la obra de uno de los fundadores de la teoría marxista de la dependencia, Ruy Mauro Marini, Patrón de reproducción del capital en Chile, (1981). Quien posteriormente desarrolla con gran rigor teórico-metodológico esta categoría es Jaime Osorio. Ver sus obras Crítica de la economía vulgar (2004); Teoría marxista de la dependencia (2016).

2 Desde su surgimiento histórico el capital arriba al mundo «chorreando sangre y lodo, por todos los poros, desde la cabeza hasta los pies». Marx, K. (2003, pág. 950). El capital, 19. ${ }^{a}$ edición, t. Iv. Siglo XxI editorial.

3 Sobre la categoría de «Patrón exportador de especialización productiva» consúltense los trabajos de Jaime Osorio (2004), en particular, cap. 4: «El nuevo patrón exportador latinoamericano»; Explotación redoblada y actualidad de la revolución (2012), en particular cap. x, «El nuevo patrón exportador de especialización productiva»; Teoría marxista... (op. cit.), en particular, «El nuevo patrón exportador de especialización productiva. Estudio a partir de cinco economías de la región».

4 La pérdida de participación del sector industrial en el PIB de la región es intensa. El análisis sobre la caída del sector industrial en el piB de Brasil, Perú, Chile, México y Colombia en los períodos 1970-1979, 1980-1989, 1990-1999, y 2000-2011, es ilustrativo: Brasil pasa de 30 a 33\% en su participación industrial, para descender al $20 \%$, y posteriormente cae al $17 \%$, respectivamente. Perú pasa del $21 \%$ al $27 \%$, cae al $17 \%$, y posteriormente disminuye al $16 \%$. El descenso de Chile va del $24 \%, 20 \%, 19 \%$ y $15 \%$, respectivamente. La participación industrial en el PIB de México cae del 23\% en los dos primeros periodos, al $20 \%$, y posteriormente cae al $18 \%$. Por último, Colombia experimenta el descenso industrial del $23 \%$ al $22 \%$, para caer al 17\%, y posteriormente disminuye al 15\%, respectivamente. Ver Asociación Nacional de Instituciones Financieras (2012).

5 Alicia Bárcena (2017, pág. 26); CEPAL (2016, pág. 73).

$6[\ldots]$ «la monopolización de valores de uso - software, conocimiento, innovación, repuestos, equipos, etc.- por las economías centrales, juega un papel clave en el intercambio desigual, junto con las consecuentes diferencias de productividad. La necesidad de compensar estas transferencias de valor mediante la superexplotación del trabajo en las economías dependientes se hace hoy más actual que nunca» (Osorio, pág. 216).

7 A este respecto, la CEPAL propone un nuevo «giro estructural», orientado básicamente por un nuevo proceso de «reindustrialización», mayor incorporación de valor agregado en commodities, una mayor diversificación de la economía, desarrollo de procesos intensivos en conocimiento, profundización del comercio intrarregional. La pregunta es si este «giro» permite resolver de modo sustantivo nuestros problemas y necesidades sociales.

8 Al hacer una descomposición por producto de las exportaciones se entiende la importancia del crecimiento de los productos básicos en nuestros países, y el incremento de su participación en la estructura exportadora.

9 En el periodo 2002-2015, las fortunas de los multimillonarios de América Latina crecieron al 21\% anual. (Comunicado, CEPAL -OXFAM, 17/03/2016).

10 De acuerdo con CePAL, en América Latina y el Caribe, el 10\% más rico concentra el 71\% de la riqueza. En contraste, solo tributa el $5,4 \%$ de su renta (ibíd.) 
11 En 2013, el 1\% de las empresas exportadoras concentra más del 70\% de las exportaciones en la región. En Brasil, con alrededor de 19 mil empresas exportadoras, el 1\% concentran el 75,8\% de las exportaciones. México, con 34.000 empresas exportadoras, el $1 \%$ concentra el 73,3\% de las exportaciones (Urmeneta, 2016).

12 Consideremos también que la economía China se encuentra en proceso de transición de su economía a un «nuevo modelo económico». Como plantea CEPAL: «La estrategia actual de desarrollo de China incluye cambiar el motor del crecimiento desde la inversión y las exportaciones al consumo privado, mediante una ampliación del ingreso disponible. Además, tiene el objetivo de aumentar la diversificación productiva del país hacia industrias intensivas en conocimiento, desarrollar los servicios, avanzar hacia una producción más limpia e incrementar la innovación, objetivos que probablemente se reforzarán en el plan quinquenal para el período entre 2016 y 2020». (CEPAL, 2015).

13 CEPAL (2016), la caída en el valor de las exportaciones y el deterioro de los términos de intercambio fueron más agudos en los países y subregiones exportadoras de petróleo y sus derivados y de materias primas.

\section{REFERENCIAS}

Amín, S. (2016, octubre 14). Entrevista. Revista electrónica Rebelión. Recuperado de http://www.rebelion. org/noticia.php?id=217929\&titular=\%22la-afirmaci\%F3n-de-la-soberan\%EDa-nacional-popularfrente-a-la-ofensiva-del-capital-\%22-

Antunes, R. (2005). Los sentidos del trabajo. Herramienta-TEL.

Asociación Nacional de Instituciones Financieras (ANIF-Colombia). (2012). El debate de la desindustrialización en Colombia. (s. d.).

Bárcena, A. (2017, abril 20). Panorama económico y social de América Latina y el Caribe ante el nuevo contexto global. CEPAL. Recuperado de https://www.cepal.org/sites/default/files/presentation/ files/170420_version_final_universidad_de_chile_-_2017.pdf

Comisión Económica para América Latina y el Caribe (CEPAL). (2016, marzo 17). La concentración del ingreso y la riqueza está en el corazón de la desigualdad en la región: CEPAL-OXFAM. Recuperado de https://www.cepal.org/es/comunicados/ la-concentracion-ingreso-la -riqueza-esta-corazon-la-desigualdad-la-region-cepal-oxfam

Comisión Económica para América Latina y el Caribe (CEPAL). (2016). Panorama de la inserción internacional de América Latina y el Caribe, 2009-2010; 2011-2012; 2015, 2016. (s. d.).

Comisión Económica para América Latina y el Caribe (CEPAL). (2018). Estudio económico de América Latina y el Caribe, 2016. (s. d.).

Comisión Económica para América Latina y el Caribe (CEPAL). (2018). Perspectivas del comercio internacional de América Latina y el Caribe, 2016. (s. d.).

Foster, B. y Magdoff, F. (2010, diciembre 11). La gran crisis financiera: tres años ya y continúa. Recuperado de www.vientosur.info

Marini, R. M. (1974). Dialéctica de la dependencia. México DF, México: Era.

Marini, R. M. (1981). Patrón de reproducción del capital en Chile. Centro de Información, Documentación y Análisis del Movimiento Obrero (Cidamo).

Marx, K. (2003). El capital, 19. a edición, t. Iv. México DF., México: Siglo XxI.

Mészaros, I. (2009). La crisis estructural del capital. Caracas, Venezuela: Ministerio del Poder Popular. Venezuela.

Osorio, J. (2004). Crítica de la economía vulgar. México DF, México: Miguel Ángel Porrúa-UAz. 
Osorio, J. (2012). Explotación redoblada y actualidad de la revolución. México DF, México: Miguel Ángel Porrúa-UAz.

Osorio, J. (2016). Teoría marxista de la dependencia. México DF, México: UAM-Ítaca.

Poulantzas, N. (1976). Las clases sociales en el capitalismo actual. México DF, México: Siglo XxI.

Sotelo Valencia, A. (2003). La reestructuración del mundo del trabajo. Itaca-UOM.

Urmeneta, R. (2016). Dinámica de las empresas exportadoras en América Latina. CEPAL. 